\title{
Interstitial Fluid
}

National Cancer Institute

\section{Source}

National Cancer Institute. Interstitial Fluid. NCI Thesaurus. Code C120839.

The extracellular fluid component found in the spaces between tissues. This excludes plasma and transcellular fluid. 